\title{
Tension pneumothorax and diffuse subcutaneous emphysema as a complication of cardiopulmonary resuscitation
}

\author{
Ahmad Sharayah, ${ }^{1}$ Dileep Unnikrishnan, ${ }^{1}$ Prem Shanker Shukla, ${ }^{1}$ Douglas Livornese ${ }^{2}$
}

'Internal Medicine, Monmouth Medical Center, Long Branch, New Jersey, USA

${ }^{2}$ Pulmonary and Critical Care, Monmouth Medical Center, Long Branch, NJ, Monmouth

\section{Correspondence to} Dr Ahmad Sharayah, drsharayah@gmail.com

Accepted 27 November 2017

\section{DESCRIPTION}

A 65 -year-old man, with a medical history of interstitial lung disease and a recently diagnosed lung adenocarcinoma with known metastases to the bone, was brought to the emergency department after an episode of cardiopulmonary arrest and resuscitation

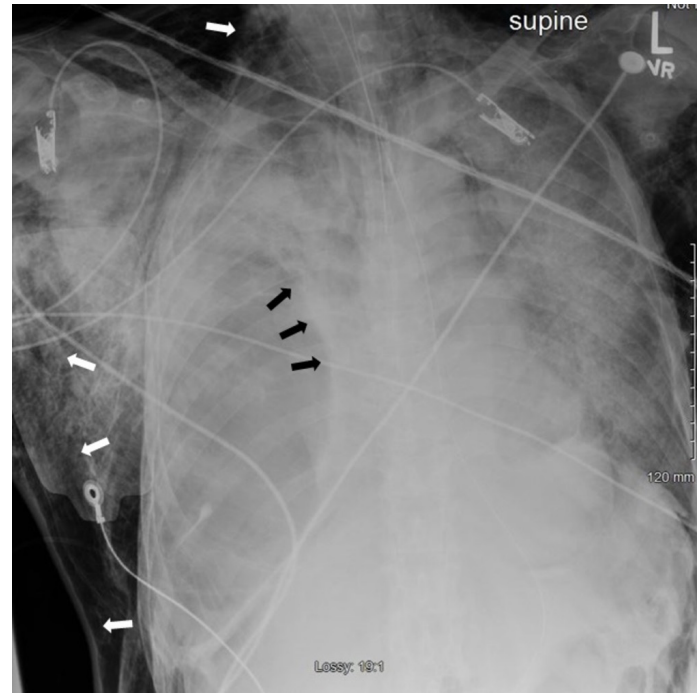

Figure 1 Hyperlucency of lung fields and absent lung markings of the right mid and lower lung fields suggestive of large right pneumothorax. ${ }^{3}$ Borders of the collapsed lungs are indicated by black arrows and subcutaneous emphysema is indicated by white arrows.

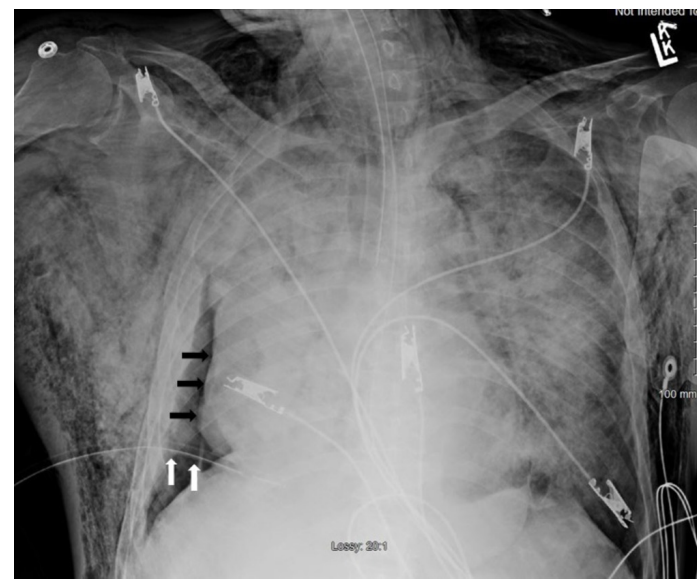

Figure 2 Partial re-expansion of the collapsed lung is seen in the figure after the insertion of the chest tube. Lung border is indicated by black arrows and chest tube is indicated by white arrows; subcutaneous emphysema is still present. by emergency medical services (EMS). The patient had a recent hospitalisation for bronchoscopic lung biopsy. It was complicated by a pneumothorax, and he was treated with chest tube insertion. The tube was removed 2 weeks prior to the current encounter and no residual leak was found. On the day of arrival, early morning, the patient's wife found him unresponsive with a temperature of 101 degrees Fahrenheit (38.3 Celsius). EMS was called and the patient was found in pulseless electrical activity. Advanced cardiac life support was initiated and he had a return of spontaneous circulation after two doses of $1 \mathrm{mg}$ epinephrine over approximately $6 \mathrm{~min}$ of cardiopulmonary resuscitation (CPR). The patient was intubated in the field and brought to the emergency department. On initial evaluation, the patient was hypotensive, for which he was started on vasopressors and aggressive intravenous fluid resuscitation. On physical exam, the patient was unresponsive, and had diffuse swelling and crepitus extending from his face down to his thighs. On chest auscultation, no breath sounds were audible on the right side.As the incidence rate of tension pneumothorax secondary to invasive lung procedures is $0 \%$ to $4 \%{ }^{1}$ a chest X-ray (figure 1) was done which showed diffuse subcutaneous emphysema and a large right lung lucency suggestive of tension pneumothorax. The diffuse granular opacities on the left side were unchanged from the previous study and represented his diagnosis of interstitial lung disease. The patient had an emergent insertion of a right-sided chest tube, to allow lung reexpansion of the lung and to imrpove ventilation, ${ }^{2}$

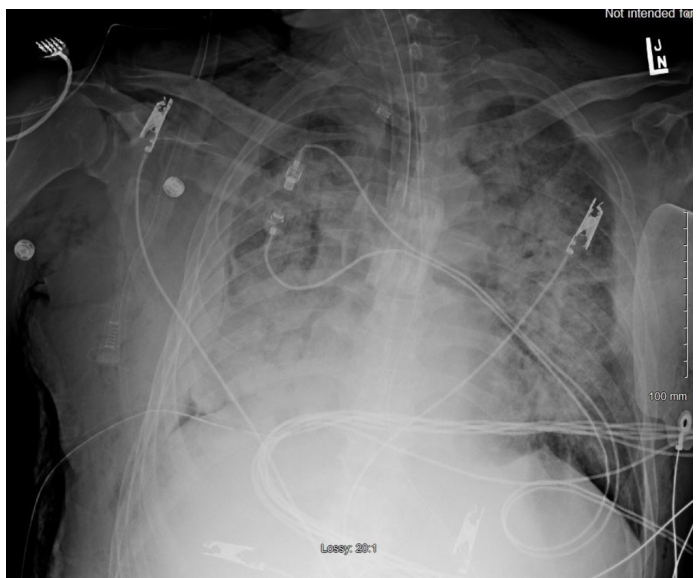

Figure 3 Almost complete re-expansion of the collapsed lung and resolution of subcutaneous emphysema can be seen in the X-ray taken 1 day after chest tube insertion. 


\section{Learning points}

- Tension pneumothorax is a potential complication among patients undergoing invasive lung procedures, such as bronchoscopic biopsy. . Due to altered lung mechanics and significantly reduced preload, tension pneumothorax causes significant respiratory and circulatory compromise if not promptly reversed. This complication should be considered in patients who underwent recent invasive lung procedures who present to the emergency department with profound hypotension, respiratory distress or both.

- Hyperlucency of lung field and absence of lung markings with delineation of collapsed lung border in chest radiograph are classical findings in a patient presenting with tension pneumothorax. However, these findings can be obscured in patients who present with hydropneumothorax or pyopneumothorax or coexisting subcutaneous emphysema. In such cases, other clinical findings such as absent lung sounds and hyper-resonance on percussion can aid in reaching the diagnosis.

- In cases of tension pneumothorax, a chest tube insertion to release the air trapped in the pleural space allows re-expansion of the lung with resultant improvement in ventilation. Ensuing reduction of intrathoracic pressure results in restoration of cardiac preload and improves cardiac output. Patients with persistent air leak or failure of lung re-expansion should be evaluated by thoracic surgery for video-assisted thoracoscopic surgery.

which drained over $1 \mathrm{~L}$ of purulent foul-smelling material. Significant air leak was noted when the chest tube was connected to the drainage system. His vital signs improved significantly after chest tube insertion. Subsequent chest X-rays, immediately after insertion (figure 2) and the next day (figure 3), showed partial re-expansion and improvement of his subcutaneous emphysema. The patient was admitted to the intensive care unit and was started on broad-spectrum antibiotics. The pus culture from the empyema grew Streptococcus anginosus and the antibiotics were narrowed down to levofloxacin. Because of the culture results, the aetiology of the patient's infection was attributed to aspiration pneumonia complicated by empyema. Aggressive CPR on the patient led to air entry to the thoracic cavity leading to the final clinical picture of pyopneumothorax and subcutaneous emphysema. In the next few days, he was weaned off vasopressor support, and his care was transitioned to a long-term acute care facility.

Contributors AS, DU, PSS and DL have been directly involved in the care of the patient and the contributions to this article.

Competing interests None declared.

Patient consent Obtained.

Provenance and peer review Not commissioned; externally peer reviewed.

(c) BMJ Publishing Group Ltd (unless otherwise stated in the text of the article) 2017. All rights reserved. No commercial use is permitted unless otherwise expressly granted.

\section{REFERENCES}

1 Leiten EO, Martinsen EM, Bakke PS, et al. Complications and discomfort of bronchoscopy: a systematic review. Eur Clin Respir J 2016;3:33324.

2 MacDuff A, Arnold A, Harvey J. Management of spontaneous pneumothorax: british thoracic society pleural disease guideline 2010. Thorax 2010;65(Suppl 2):ii18-31.

3 Seow A, Kazerooni EA, Pernicano PG, et al. Comparison of upright inspiratory and expiratory chest radiographs for detecting pneumothoraces. AJR Am J Roentgenol 1996;166:313-6.

Copyright 2017 BMJ Publishing Group. All rights reserved. For permission to reuse any of this content visit

http://group.bmj.com/group/rights-licensing/permissions.

BMJ Case Report Fellows may re-use this article for personal use and teaching without any further permission.

Become a Fellow of BMJ Case Reports today and you can:

- Submit as many cases as you like

- Enjoy fast sympathetic peer review and rapid publication of accepted articles

- Access all the published articles

- Re-use any of the published material for personal use and teaching without further permission

For information on Institutional Fellowships contact consortiasales@bmjgroup.com

Visit casereports.bmj.com for more articles like this and to become a Fellow 